\title{
Mechatronics and bioinspiration in actuator design and control
}

\author{
J.L. Pons, A. Forner-Cordero*, E. Rocon and J.C. Moreno \\ Bioengineering Group, Consejo Superior de Investigaciones Cientificas, CSIC, Madrid, Spain \\ (Received 29 February 2008; final version received 1 July 2008)
}

\begin{abstract}
Actuators are components of motion control systems in which mechatronics plays a crucial role. They can be regarded as a paradigmatic case in which this mechatronic approach is required. Furthermore, actuator technologies can get new sources of inspiration from nature (bioinspiration). Biological systems are the result of an evolutionary process and show excellent levels of performance. In this paper, we analyse the actuator as a bioinspired mechatronic system through analogies between mechatronics and biological actuating mechanisms that include hierarchical control of actuators, switched control of power flow and some transduction principles. Firstly, some biological models are introduced as a source of inspiration for setting up both actuation principles and control technologies. Secondly, a particular actuator technology, the travelling wave ultrasonic motor, is taken to illustrate this approach. Eventually, the last section draws some conclusions and points out future directions.
\end{abstract}

Keywords: actuators; bioinspiration; biomechatronics; mechatronics

\section{Introduction: mechatronics and actuators}

The term 'mechatronics' was coined in Japan in the mid1970s to denote the engineering discipline dealing with the study, analysis, design and implementation of hybrid systems comprising mechanical, electrical and control (intelligence) components or subsystems (Pons 2008).

Mechatronic systems are in many instances synonymous with motion control systems. Actuators can be regarded as key elements in motion control systems. The mechatronic approach to systems development presents distinctive characteristics. A very interesting feature of mechatronic systems is the combination of functions in the same component. When dealing with actuators, we are especially interested in the possible combination of control, sensing and actuation functions in a system, that is, intelligent concomitant sensing and actuation (Pons 2005).

The combination of functions using mechatronic integration of disciplines in the design of actuators has clear functional benefits. The miniaturization of systems is another distinctive characteristic of a mechatronic approach and can be seen as a direct consequence of this combination of functions. Yet, the paradox of opposite rationales in the design of sensors and actuators must be addressed (BuschVishniac 1998). Both sensors and actuators are transducers. On the one hand, sensors are transducers in which a low level of energy conversion is desired, that is, in this way the measured variable is not influenced by the measuring process. On the other hand, actuators require a high level of power conversion, that is, the mechanical state imposed by the actuator is, in this way, not influenced by load or external perturbations.

An example is the biological muscle. It contains an actuation (contractile elements) with included sensors, such as the muscle spindles (which sense the length changes and its rate of change) and the Golgi tendon organs (which sense the forces). These sensors can be tuned to respond adaptively depending on the situation (gamma activation). The reflexes implemented with these sensors, along with the intrinsic properties of muscle, result in a robust system that is not unstable despite of the relatively long transmission delays (Prochazka et al. 1997).

The mechatronic combination of functions on an actuator has been pursued intensively during the past years. Kuhnen and Janocha (1994) estimated the load on a piezoelectric actuator in order to compensate for its disturbing effect during actuation. Also in the context of piezoelectric actuators, Dosch et al. (1992) implemented a collocated and concomitant position and velocity feedback on an actuator. Simmilar approaches have been proposed for electromagnetic actuators, shape memory alloy actuators, magnetostrictive actuators and electroactive polymer actuators (Pons 2005).

\section{Actuators: the paradigm of mechatronics}

The concept of an actuator as a true mechatronic system is illustrated with the example of a resonant piezoelectric drive. A piezoelectric ceramic is characterized electrically by a capacitive load whenever it is out of resonance and a

*Corresponding author. Email: aforner@iai.csic.es 


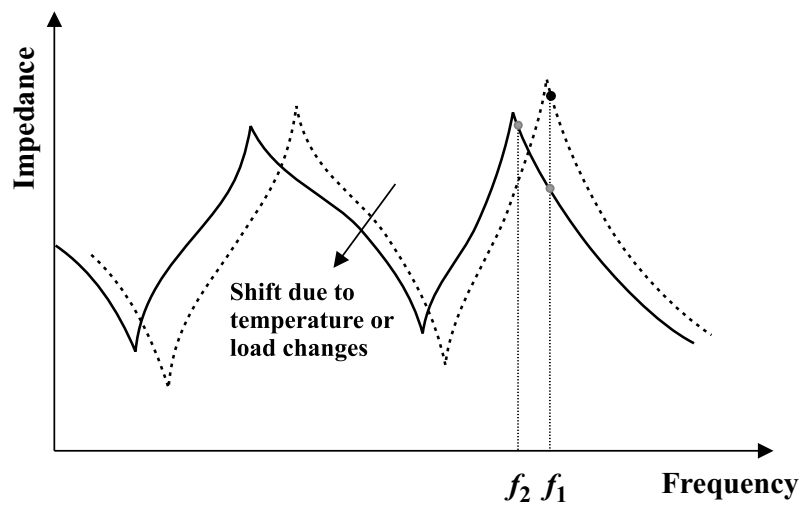

Figure 1. Effect of temperature or load fluctuations on the resonance characteristics of piezoelectric actuators and corresponding modification of the operating point.

resistive electrical load when it is close to resonance (local minimum in mechanical impedance) or antiresonance (local maximum in electrical impedance). Piezoelectric resonators are driven close to their resonance or antiresonance frequencies.

In actuators of this type, an applied external load or temperature change leads to a shift in the resonance frequency (see Figure 1). If this is not compensated for the operating point of the piezoelectric drive is generally not perfectly tuned. Note the relative position of the original operating point (black dot at $f_{1}$ in Figure 1 ) with respect to the resonance as compared with the new relative position (grey dot at $f_{1}$ ).

With a mechatronic approach, a self-tuning electrical driver can be designed that will track any possible fluctuation in the resonance characteristics of the actuator, and thus the new operating point will be tuned to the new resonance curve (see grey dot at $f_{2}$ in Figure 1). In so doing, the phase between voltage and current can be used as an indicator of the electrical impedance of the actuator. This can then be used to close the loop, for instance, by means of a phase locked loop (PLL).

The resonant piezoelectric actuator as described above includes an actuator system (the voltage-driven piezoelectric resonator), a sensor system (monitoring the phase lag or impedance condition at the input port) and a disturbance rejection control system (the PLL drive).

Engineering disciplines have ever looked to nature as a source of inspiration. Several million years of evolution has seen living creatures progress to their current state. Engineering has very often taken models from nature and mimicked biological structures.

Mechatronics as an engineering discipline may also benefit from seeking nature as a source of inspiration. As noted earlier, mechatronic systems are in most cases equivalent to motion control systems. As such, the motor control structure of upper mammalians is a perfect model in which to find inspiration.
This paper addresses the analysis of intelligent actuators as mechatronic systems and the role of bioinspiration in the conception of these actuators. In this context, biological actuators usually show simultaneous sensing, actuation and control. The actuator itself can be shown to exhibit the same mechatronic characteristics as a motion control system. This means that the actuator can be analysed as a mechatronic system and it will benefit from the intrinsic cross-fertilization between engineering disciplines (Reynaerts et al. 1998). In the next section, nature is presented as a source of inspiration for the mechatronic design and implementation of actuators. This is done by introducing a few examples of biological models both for control strategies and for actuation principles. Section 3 addresses the bioinspired mechatronic design of travelling wave ultrasonic motors (TWUMs). The last section draws some concluding remarks.

\section{Mechatronics and biomimesis}

This section introduces biological models for the hierarchical control of actuators, the switched control of power flow to actuators and some actuator principles.

\section{Hierarchical motor control in mammalians as a model}

Hierarchical control schemes are common in motion control systems. There is sufficient evidence to support the view that the structure of the motor control system in mammals is hierarchically organized. This organization allows an excellent performance of both human and non-human primates in manipulation tasks. This performance in manipulation comprises, among other functions, superb response to disturbance, for example, increased prehensile force following slippage of grasped objects and perfect modulation of upper-limb (impedance controlled) interaction with the environment.

In these particular manipulative tasks, feedback control schemes involving structures in the central nervous system (CNS) do not seem feasible. In fact, the shortest loop delay involving neural transmission from skin receptors to the CNS and back is in the region of $100-150 \mathrm{~ms}$. If these feedback loops involve computing at the brain level (for instance, in visual feedback operations), the loop delay can reach up to 200-250 ms. With loop delays of this magnitude, the effectiveness of feedback modulation of impedance or the response to disturbance would be very much compromised.

Hierarchical control schemes mimicking the structure of the human motor control system are a common approach. Figure 2 shows a schematic representation of such a hierarchical control scheme. An upper-level task planner is in charge of sending motion commands (reference trajectories) to low-level controllers. The low-level controllers 


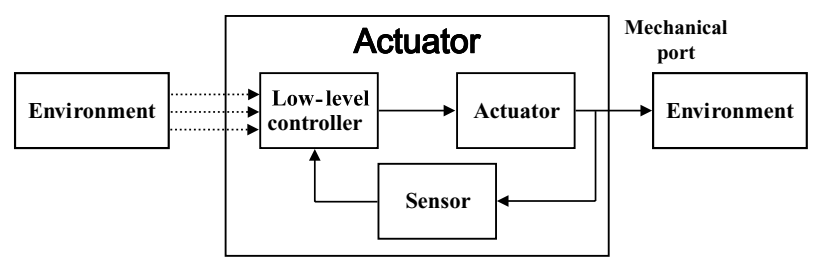

Figure 2. Hierarchical representation of the human motor control system.

interact with the plant through sensors and actuators (including the corresponding impedance matching stages). As seen from the upper-level controller, the process is an open loop.

This process could be illustrated in the control strategy for tremor suppression implemented in the exoskeleton WOTAS (Rocon et al. 2007). The behaviour and contribution of each joint in the upper-limb tremor is such that in most patients the tremor movement 'displaces' along the kinematic chain of the arm when its effects are attenuated by applying a biomechanical load on one of the joints. This aspect led to devising active and hierarchically independent control strategies in each joint. Accordingly, if the cancellation of the tremor in one of the joints increases the tremor in the other joint, the algorithm responsible for controlling the adjacent joint will identify the increased tremor and try to reduce the tremor generated by coupling the upper-limb joints, thus showing the distributed hierarchical control typical from biological systems. The aim is thus that the active behaviour of tremor reduction in each joint reaches equilibrium, thereby decreasing the coupling effects of the upper-limb joints.

\section{Switching control of muscle contraction as a model to modulate the input power in actuators}

The human musculo-skeletal system is driven through switched techniques. Motor stimuli reach the various different muscles through motoneurons. Each muscular stimulus leads, in the first instance, to muscle contraction followed by relaxation. The time constants of the contraction and relaxation processes are very different. The muscle contraction time constant is much lower than the relaxation time constant; as a result, the musculo-skeletal system responses during contraction and relaxation exhibit different dynamics (see the different dynamics $-\tau_{C}$ for contraction, $\tau_{R}$ for relaxation, where $\tau_{C} \ll \tau_{R}$-in a muscle twitch inset in Figure 3).

If repeated stimuli reach the muscle prior to total relaxation, summation occurs and the result is increasing contraction. Overall muscle contraction is a combination of increased contraction of individual fibres due to summation and increased recruiting of additional motoneurons, and consequently muscle fibers. See Figure 3 for a schematic representation of the switched control of muscle contraction.

In addition to position control of the human musculoskeletal system, there is sufficient evidence to believe that the modulation of the motor activity in antagonistic muscles is one of the mechanisms that mammalians use to modulate the impedance around an equilibrium position (Hogan 1985).

Switching techniques as an approach to modulation of the flow of energy in actuators are of particular interest when actuators exhibit different dynamics in both directions (such as in the case of muscle contraction-relaxation). This is generally true for thermal actuators and, in particular, shape memory alloy actuators. In these systems, the time

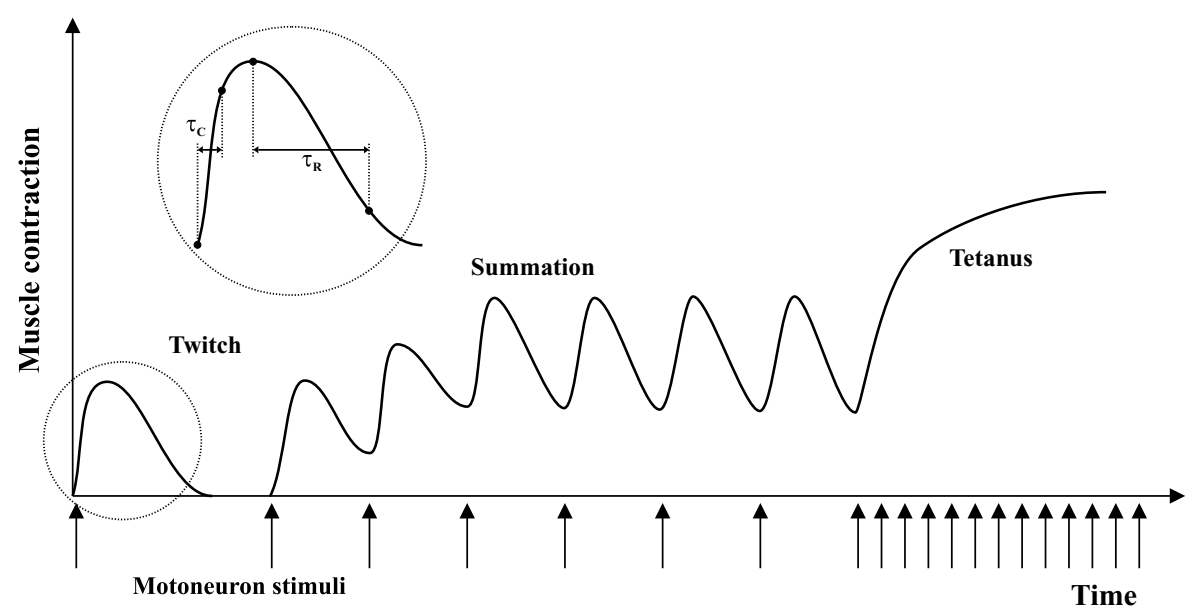

Figure 3. Biological model switching techniques to modulate the flow of energy in actuators. 


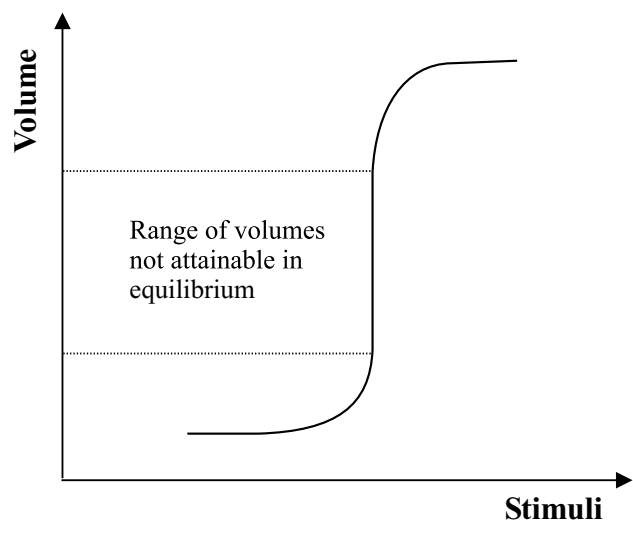

Figure 4. Discontinuity in volume-phase transformations leading to non-attainable mechanical states.

constant for the heating process is generally much lower than for the cooling process (which is limited by thermal inertia and heat dissipation).

Wherever a discontinuity occurs in the change in length of an actuator, switching techniques may be the only possible solution for accurate positioning tasks. The discontinuity, as depicted in Figure 4, leads to mechanical states that are not attainable in equilibrium. This is, for instance, the case of some electroactive polymer actuators, in particular, polymer gel actuators (Pons 2005). In such a case, switching techniques can maintain the mechanical state without equilibrium within the margin of mechanical state error allowed by the application (Mitwalli 1998).

\section{Actuation principles based on biological models}

The previous two examples of biomimesis are more closely related to the way actuators can be driven and how the energy flow is modulated. Nature is full of models for establishing actuation principles. Here, we briefly describe two locomotion models that inspired the development of the socalled inchworm actuators and travelling wave linear and rotational ultrasonic motors.

The first model is the locomotion process of some earth worms as depicted in Figure 5. This locomotion process has two modes of movement, one consists of fixing alternatively the front and rear legs to the ground and the other consists of elongating and contracting the intermediate segments of the worm. This locomotion is split in two cycles: In the first cycle, the rear legs are fixed to the ground while the front legs are free. Then, the intermediate segment elongates. In the second cycle, the front legs are fixed while the rear ones are free and the intermediate segments of the worm contract. Both cycles are nested to provide the locomotion.

The same principle is followed in the development of inchworm piezoelectric motors. Here, three independent piezoelectric ceramics (see schematic view inset in

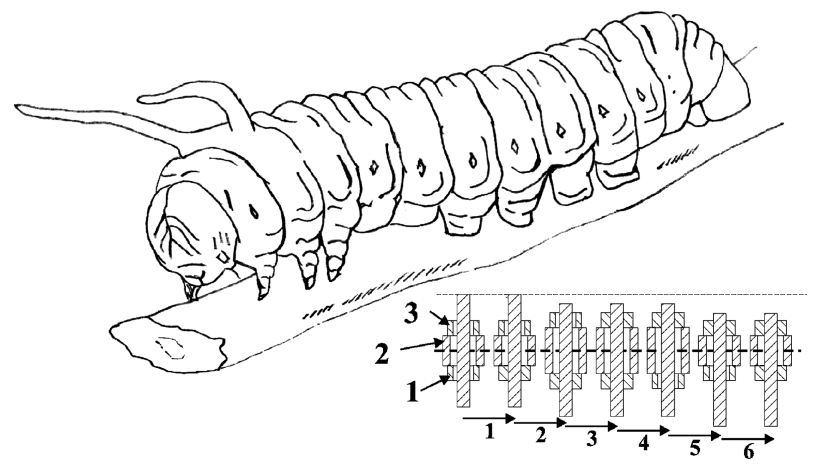

Figure 5. Biological model for inchworm piezoelectric actuators.

Figure 5) are used to mimic the operation of rear and front legs (ceramics 1 and 3) and the intermediate segments (ceramic 2). The piezoelectric actuators 1 and 3 are driven according to the first cycle so that they clamp the rotor (displacer) alternately. The piezoelectric actuator 2 is driven according to the second cycle, mimicking the elongation and contraction of the intermediate segments of the worm.

The second locomotion principle is found in some millipedes and centipedes (see Figure 6). The motion of the different legs is coordinated to produce an approximate sinusoidal pattern in both the elevation and the forwardbackward movement. These sinusoidal movements in perpendicular directions produce an elliptic movement of each leg. This elliptic movement (which is implemented in successive legs with a small delay) provides incremental traction to the millipede.

The same principle is exploited in TWUMs. We analyze this in more detail in Section 3.

\section{A system-level biomimetic approach: human knee joint behaviour during gait}

Knee-ankle-foot orthoses (KAFOs) are systems used to restore human gait, providing stability during stance phase. Some orthotic systems are based on providing a fixed stiffness at the knee joint. However, the knee has different requirements during swing or stance phases of gait. The knee has to be flexed during initial swing and extend on time for foot contact. During stance, it should provide support for the body weight.

A concept of actuator for a KAFO was developed on the basis of the biomechanical data. The actuator is conceived to provide mechanical means to reproduce the normal kinematics during human gait at the joint level. Lower limb joints behaviour was approximated by elastic means. The rationale of the design process considered the functional aspects and aimed at a lightweight solution with low power demand (see Figure 7). 


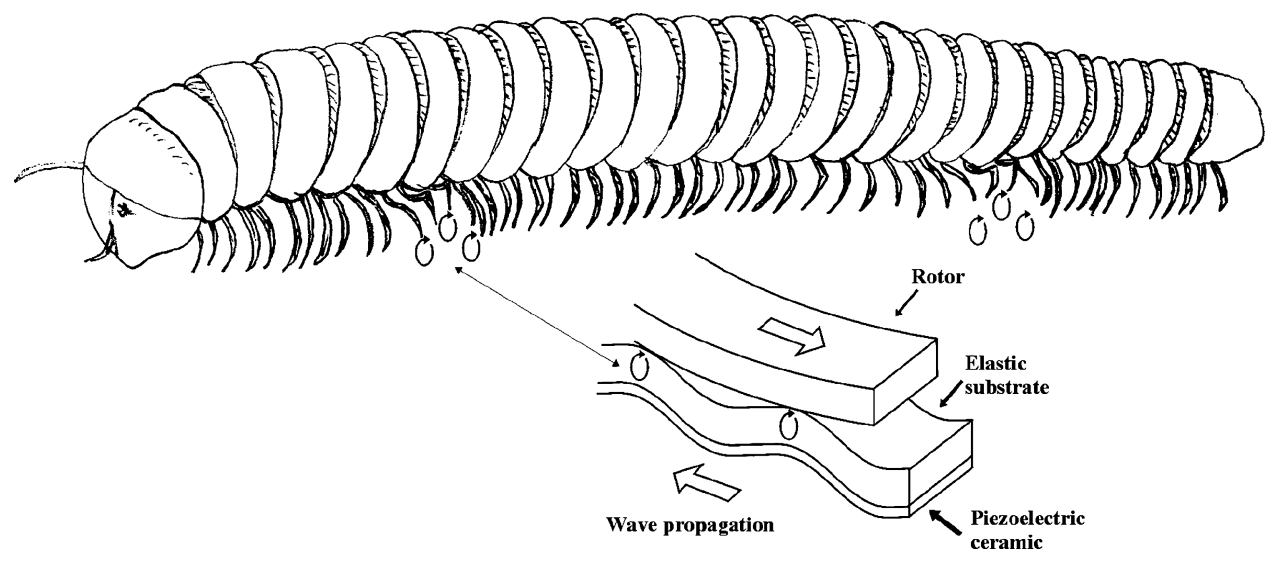

Figure 6. Biological model for travelling wave ultrasonic linear and rotational motors.

The solution consisted of using two springs to simulate the knee function during gait with an orthosis. During the initial swing phase, a low stiffness spring allows for the knee flexion due to inertia. At mid-swing, this spring recovers length to assist the knee extension required before foot contact and at its maximal elongation switches to a high stiffness spring. This mechanism was simulated on a computer and tested on healthy subjects and in patients with lower limb weakness, more specifically, post-poliomyelitis syndrome.

The knee function was approximated by two linear springs acting at different phases of the gait cycle. It sim- ulated knee function during gait, given the inertial parameters of the leg and a limited range of gait speeds and step lengths. It was found that the ratio between both springs must be 10 with a minimal low stiffness of 0.07 $\mathrm{N} / \mathrm{mm} \cdot \mathrm{kg}$.

Therefore, the knee moment and the knee joint angle can be locally approximated by a linear relation as given by the two springs. However, these stiffnesses must be modulated depending on gait speed and, of course, inertial parameters of the leg.

The tests demonstrated that subjects felt more comfortable and safe with a design that considers the biomimetic

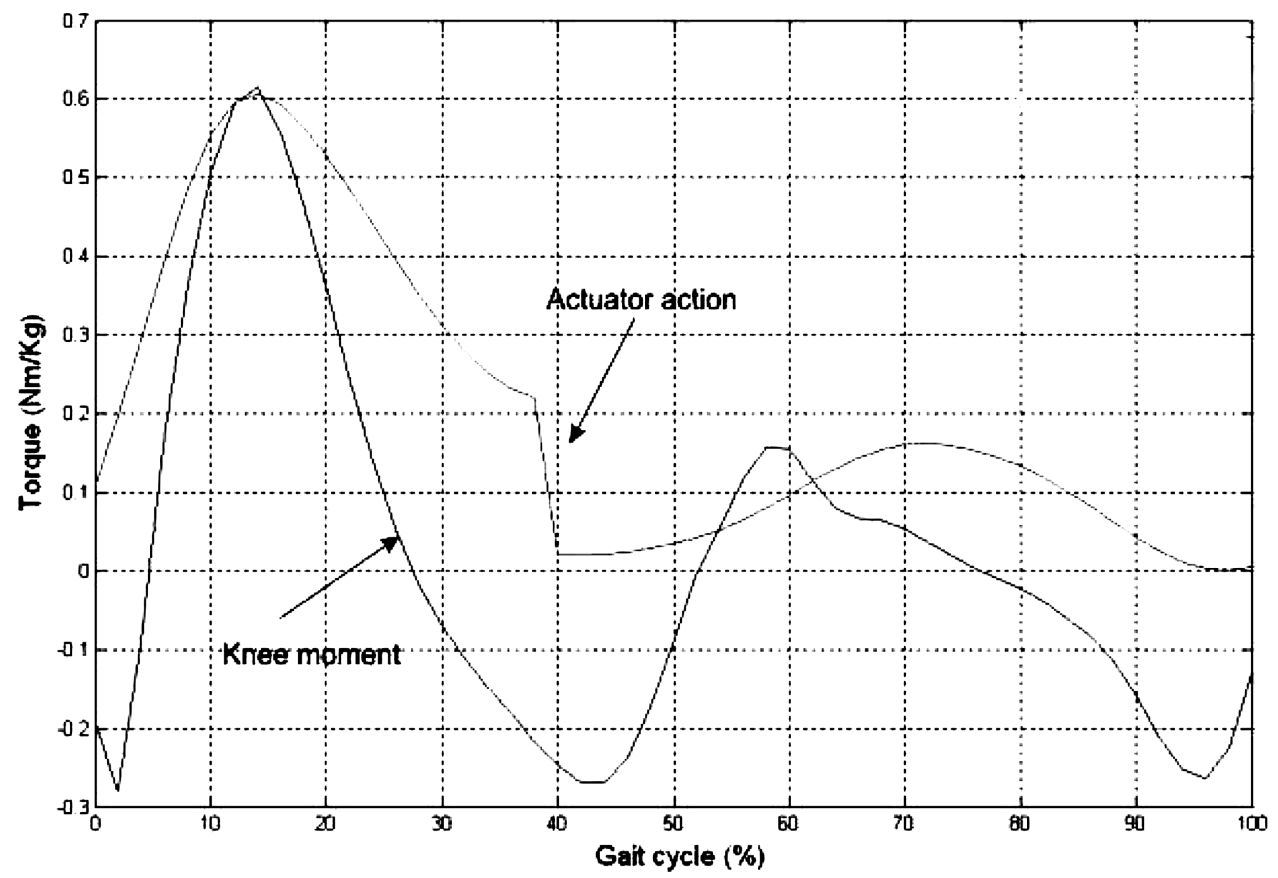

Figure 7. Comparison of knee and actuator torques during the gait cycle. The abrupt transition in the actuator torque corresponds to the change of spring stiffness. 

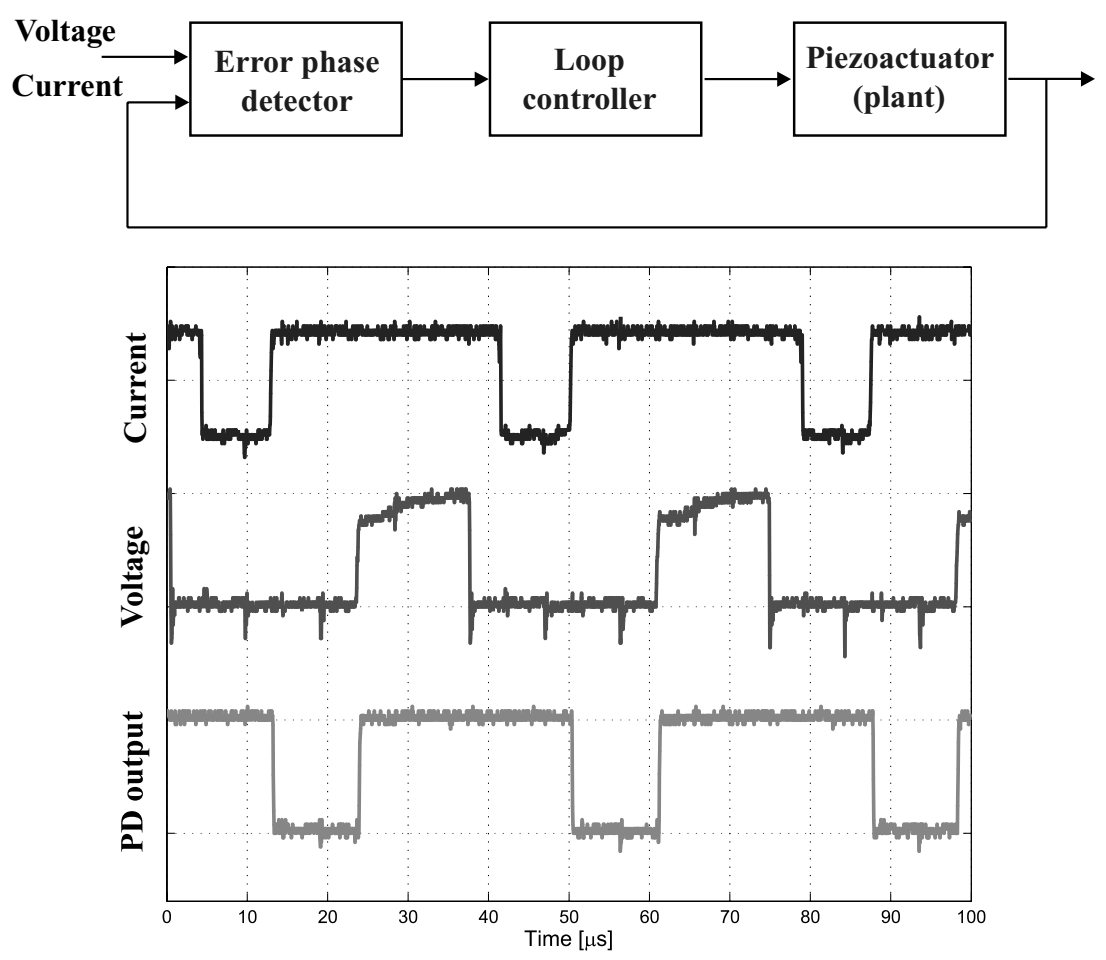

Figure 8. Perturbation rejection loop for a TWUM in which sensing and actuation functions are combined.

approach to simulate the required function. These results are detailed elsewhere, in Cullell et al. (2008)

\section{TWUMs: a bioinspired mechatronic approach}

TWUMs are a good example to illustrate the bioinpired mechatronic approach to develop actuators. The principle of actuation is inspired in the locomotion principle of millipedes as previously introduced. In TWUMs, a hierarchical control approach can be implemented in which a high level control strategy seeks for position, velocity or force control while a low-level fast loop looks for intelligent perturbation (load changes, temperature variations) rejection based on simultaneous sensing. Power input control can be easily regulated by means of switched techniques in combination with electromechanical load matching. The next section elaborates on this approach.

\section{Biomimetic actuation principle}

TWUMs comprise a stator and a rotor. The stator is an electromechanical composite (a driving piezoelectric ceramic on a metal substrate) that is driven close to resonance. The principle of operation consists of the friction transmission of microscopic elliptical oscillations between the stator and the rotor (see the schematic view inset in Figure 6).

To do so, a travelling wave is excited in the stator by means of the piezoelectric ceramic. When such a wave takes place, a number of points at the stator-rotor interface describe elliptic trajectories in phase. This travelling wave propagates in a counter-rotation manner. This is exactly the situation found in the locomotion principle of millipedes.

\section{Bioinspired hierarchical control and switched input power control}

Actuators are often hierarchically included in motion control systems. Under this approach, a trajectory planner sets a position, velocity or force reference and low-level controllers implement close loop control on these set points. In so doing, low-level controllers must implement intelligent perturbation rejection loops. This is also the case with TWUMs.

These motors are driven at antiresonance in which natural amplification is used to enable friction transmission of elliptic oscillations to the rotor. This makes TWUMs very sensitive to shifts in resonance characteristics due to external perturbations, that is, load changes, temperature fluctuations, etc.

The electrical impedance of piezoelectric ceramics is resistive in the vicinity of resonance and antiresonance and capacitive for out of resonance. Because of the electrical impedance, that is the ratio of driving voltage to current drawn, the actuator can be set to operate as a sensor by analyzing the phase lag between voltage and electrical current. Close to antiresonance, this phase lag vanishes. For 
out of resonance, the phase lag takes voltage and current to quadrature.

By using a phase detector (PD) and a voltage controlled oscillator (VCO), an intelligent resonance tracking loop can be implemented around the TWUM. This is done by combining actuation and sensing functions on the came component. This mechatronic approach results in efficient, compact and intelligent drives. Figure 8 shows the schematic view of such a perturbation rejection loop and the voltage, current and PD output signals when the actuator is out of resonance.

The control structure in the centipedes seems to be composed of a chain of central pattern generators (CPGs). These are groups of neurons that can oscillate independently at higher levels or sensory input. During normal functioning, the CPG behaviour is modulated by sensory input as well as inputs from other CPG or higher neural levels. In this way, it is possible to obtain a chain of coupled oscillators that are able to control different types of gaits (Sigvardt and Miller 1998). This would be an example of model available in nature that could be exploited for the control of TWUMs.

Taking further the bioinspired design of control approaches for TWUMs, a switched input power control strategy can be implemented. TWUMs are to be driven in resonance, therefore the fundamental frequency of the input signal (voltage signal in Figure 7) must be the resonance frequency of the stator. If a switched signal is used, we can take advantage of digital electronics to drive the actuator. In this approach, the VCO sets the control switched signal tuned to the resonance frequency. For optimal efficient control, a proper selection of duty cycle (duty cycle of $1 / 3$ for the voltage signal in Figure 7) results in simplified power electronics as harmonics are reduced and parasitic resonances are removed (Schaaf and van der Broeck 1995).

\section{Conclusion}

This paper addressed the issue of mechatronic and bioinspired conception of actuators. Actuators have been described as key elements in motion control systems. We have seen, through a few examples, how the different aspects of mechatronics (intelligence, sensing and actuation) can be inspired by biological models.

Conventional actuator technologies are clear examples of bioinspired mechatronic approaches, for instance the case of a PWM controlled DC electromagnetic motor.
However, this will become more and more apparent as new actuators for advanced motion control systems are pursued.

Recently, some electroactive polymer actuator (EAPs) are being used to develop so-called 'artificial muscles.' Also these EAPs and other technologies are finding application in domains where the boundary between artificial systems and biological structures vanishes (Smela and Gadegaard 2001; Pons 2005). We will most likely see an increasing focus on bioinspired mechatronic approaches not only in the context of actuators but also, in general, in compliant advanced mechatronic systems.

\section{References}

Busch-Vishniac IJ. 1998. Electromecanical sensors and actuators, Berlin: Springer.

Cullell A, Moreno JC, Rocon E, Forner-Cordero A, Pons JL. 2008. Biologically based design of an actuator system for a kneeankle-foot orthosis. Mech Machine Theory.

Dosch JJ, Inman DJ, García E. 1992. A self-sensing piezoelectric actuator for collocated control. J Intell Mater Syst Struct. 3:166-185.

Hogan N. 1985. Impedance control: an approach to manipulation (Part II - Implementation). J Dyn Syst Measurement Control. 107:8-16.

Kuhnen K, Janocha H. 1994. Compensation of the creep and hysteresis effects of piezoelectric actuators with inverse systems. Actuator, 98:309-312.

Mitwalli AH. 1998. Polymer gel actuators and sensors. [Ph.D. Thesis]. Massachusetts Institute of Technology.

Pons JL. 2005. Emerging actuator technologies: a micromechatronic approach. Chichester (UK): John Wiley \& Sons.

Pons JL. 2008. Wearable robots: biomechatronic exoskeletons. Chichester (UK): John Wiley \& Sons.

Prochazka A, Gillard D, Benett DJ. 1997. Positive force feedback control of muscles. J Neurophysiol. 77(6):3226-3236.

Reynaerts D, Peirs J, Van Brussel H. 1998. A mechatronic approach to microsystem design. IEEE/ASME Trans Mechatronics. 3(1):24-33.

Rocon E, Belda-Lois JM, Ruiz AF, Manto M, Moreno JC, Pons JL. 2007. Design and validation of a rehabilitation robotic exoskeleton for tremor assessment and suppression. IEEE Trans Neural Syst Rehabil Eng. 15(3):367-378.

Schaaf U, van der Broeck H. 1995. Piezoelectric motor fed by a PLL-controlled series resonant converter. Paper presented at: Proc ENE, 95 pp. 3845-3850. European Conference on Power Electronics and Applications, September 19-21 (1995), Sevilla, Spain.

Smela E, Gadegaard N. 2001. Conjugated polymer actuators for biomedical applications. J Phys Chem B. 105:9395.

Sigvardt KA, Miller WL. 1998. Analysis and modelling of the locomotor central pattern generator as a network of coupled oscillators. Ann NY Acad Sci. 16(860):250-265. 

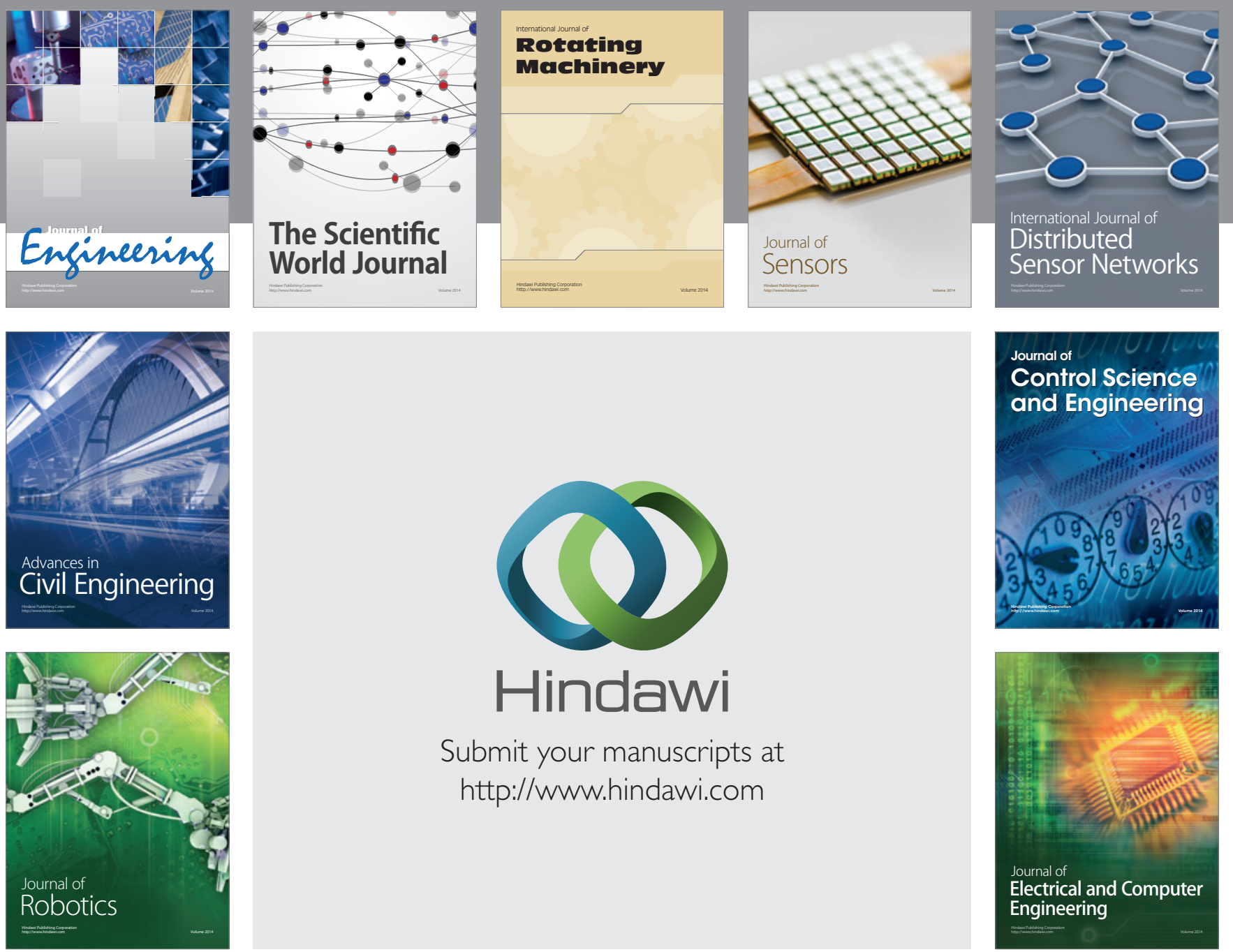

Submit your manuscripts at

http://www.hindawi.com
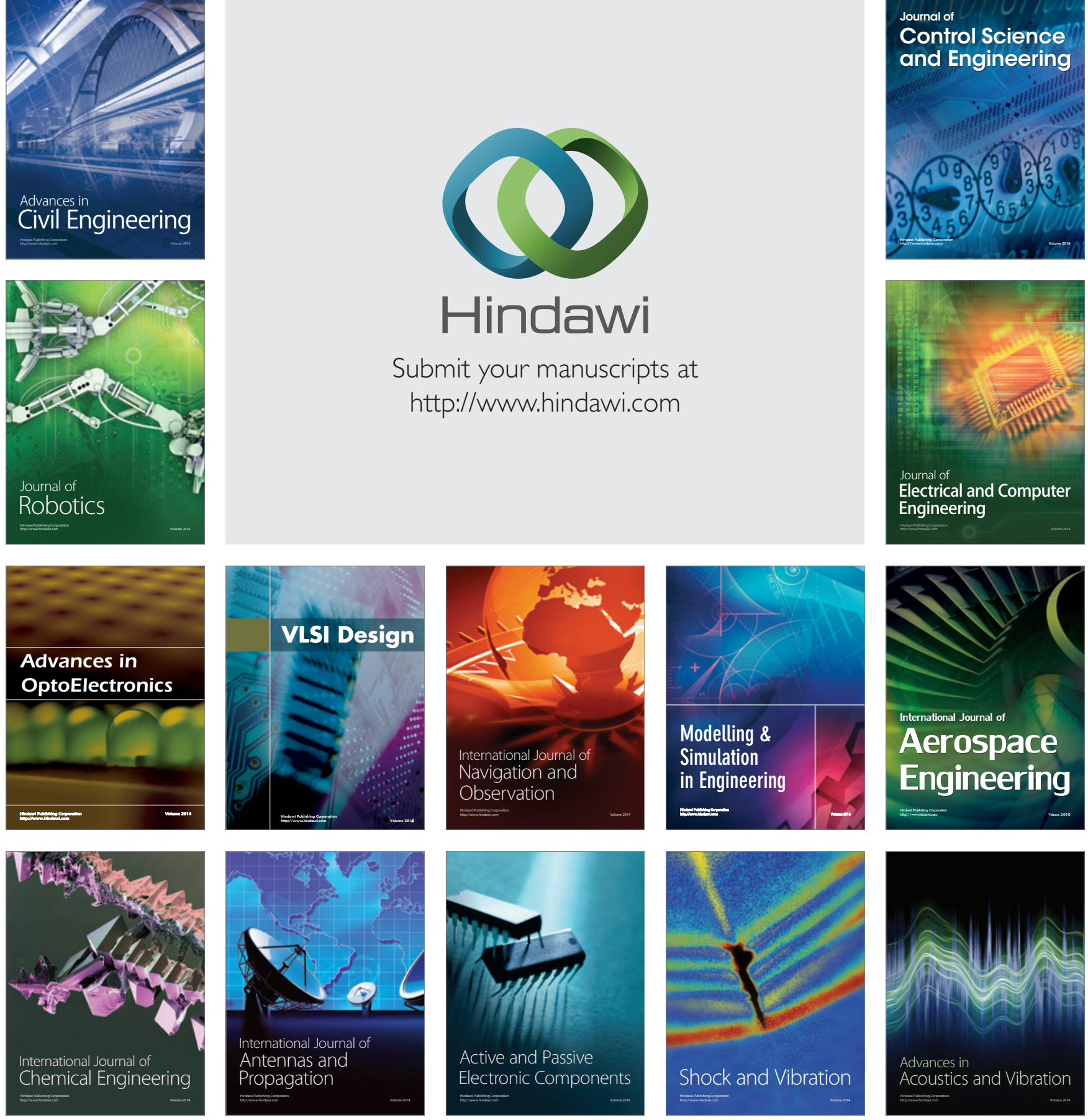